\title{
Kurze Betrachtungen über die Gruppe der Edelfalken.
}

\section{Von Carlo Freiherr von Erlanger.}

In der Zeitschrift „Aquila“ VIII. 1902 bespricht Kleinschmidt eingehend den Formenkreis von Falco Hierofalco oder Falco islandus. Ich verwende lieber letztere Bezeichnung, da ich vorerst nicht den Grund einsehe, warum Verfasser den Genusnamen "Hierofalco" in dieser trinären Zusammensetzung benutzt, wodurch nur noch eine neue Veränderung in die Nomenklatur kommt, über welche sich die Ornithologen immer noch nicht einig sind. In letzter Zeit neigen ja schon mehr zur Ansicht, dass die trinäre Nomenklatur die zweckmässigste ist, um wirklich verwandte und sich vertretende Arten zu kennzeichnen, und hoffentlich werden sich auch noch mehr Anhänger zu der Auffassung der Formenkreise oder Ringe der einzelnen Arten bekennen, welche gewissermassen aus Unterabteilungen, den zoogeographischen Formen bestehen, welche dann trinär benannt werden müssen.

K leins ch midt spricht von zwei Formenkreisen, dem von Falco Hierofalco, zu welchem er - 11 geographische Formen rechnet:

1. Falco Hierofalco islandus (Brünn.)

Verbr: Island, Grönland, Labrador, arctisches Amerika, Sibirien.

2. Falco Hierofalco gyrfalco (L.)

Verbr: Skandinavien [? Nordrussland.]

3. Falco Hierofalco uralensis (Menzbier)

Verbr: Ural.

4. Falco Hierofalco sacer (Gm.)

Verbr: Donaugebiet, Wolgagebiet, Trans.-Kaspisches-Gebiet.

5. Falco Hierofalco mexicanus (Schlegel)

Verbr: Mexiko und südliche Staaten von Nord-Amerika.

6. Falco Hierofalco feldeggi (Schleg. u. Susemihl)

Verbr: Balkanhalbinsel, Italien.

7. Falco Hierofalco erlangeri Kleinschmidt.

Verbr: Tunesien (Atlasländer).

8. Falco Hierofalco tanypterus (Schlegel)

Verbr: Nilländer, Abyssinien, Nubien, Aegypten.

9. Falco Hierofalco biarmicus (Tem.)

Verbr: Süd-Afrika.

10. Falco Hierofalco juggur (Gray)

Verbr: Indien.

11. Falco Hierofalco lorenzi (Menzb.)

Verbr: Central-Sibirien.

Neben diesem Formenkreis erwähnt er den von Falco peregrinus $(\mathrm{Gm}$.), dessen Formen nichts mit denen des Islandsfalken zu tun haben, nach Kleinschmidt den Formen von Falco Hierofalco. In den Formenkreis der blaunackigen, blauköpfigen Wanderfalken rechnet er vorerst (siehe pag. 43) auch den rotnackigen Falco barbarus und fasciinucha. Sollten wir es mit diesen rot- 
nackigen Falken nicht noch mit einem 3. Formenkreis zu tun haben, eine offene Frage?

Das Verbreitungsgebiet (gerade in den Mittelmeerländern) der rotnackigen und blaunackigen Edelfalken wäre sonst ein zu beschränktes.

Ausserdem finden sich doch zwischen den einzelnen Formen ein und desselben Formenkreises Übergänge; in zwei an einander grenzenden geographischen Gebieten sehen sich die sich vertretenden Formen ähnlich, z. B. „biarmicus" - „tanypterus" „,feldeggi" - ,erlangeri" im Gegensatz zu „,biarmicus" - „islandus". Hat man die Zwischenformen dabei, so sieht man jedoch sofort die Verwandtschaft dieser beiden Extreme.

Wie würde man sich aber dementsprechend folgende Tatsache erklären, wenn man die zoogeographische Lage der Verbreitungsgebiete berücksichtigt.

In Südostafrika, Tanganyikagebiet der rotnackige Falco fasciinucha Rchn. Neum., daran angrenzend, im Kongo, Deutsch- und British-Ost-Afrika wiederum ein anderer Wanderfalke mit völlig blauer Oberseite und blauem Nacken, und letzterem sehr ähnlich eine dritte Art auf Madagascar. Sowohl aus Deutsch-Ost-Afrika, als auch aus Madagascar liegen mir während der Brutzeit gesammelte adulte Exemplare vor. Dann wieder in Nord-Ost-Afrika, Arabien, der dem Falco fasciinucha so nahe stehende $\boldsymbol{F}$ alco barbarus arabicus Erl. Leider ist das bis jetzt vorliegende Material zumal an authentischen Brutvögelu zu gering, und darf die vorliegende Arbeit nur als Stückwerk, als eine Pionier-Arbeit für spätere Forschungen angesehen werden. Meiner Ansicht nach haben diese beiden, in Ost-Afrika und auf Madagascar als Brutvögel vorkommenden Wanderfalken gar nichts mit den andern Arten aus Süd-Ost und Nord-Ost-Afrika zu tun. Wir haben es, wie gesagt, mit Falken aus verschiedenen Formenkreisen zu tun, welche nebeneinander herlaufen. Ich kann mich daher nicht mit dem Gedanken befreunden, die blaunackigen und rotnackigen Edelfalken als zu je einem Formenkreis gehörig anzusehen. Es sollen sich vertretende Verwandte sein? Niemals!!

Ebenso halte ich es für ausgeschlossen bei Raubvögeln, die von andern Vögeln leben, deren Verbreitungsgebiet naturgemäss ausgedehnter sein muss und infolge ihrer Flugfertigkeit auch ist, dass in Marokko ein rotnackiger und blaunackiger Edelfalke (es liegen mir Brutexemplare vor) als Verwandte anzusehen, geographisch getrennt leben. Es sind zwei Falkenarten, welche nichts mit einander zu tun haben.

Meiner Ansicht nach haben wir also ausser dem von Kleinschmidt in "Aquila" behandelten Formenkreis von Edelfalken noch zwei andere, nämlich den von Falco barbarus L. und Falco? peregrinus $\mathrm{Gm}$.

Aus dem Formenkreis von Falco barbarus L. ex. Barbaria sind mir folgende Formen bekannt: 
Kurze Betrachtungen über die Gruppe der Edelfalken.

Falco barbarus barbarus L.

Ägypten, Nubien (Nilgebiet).

Falco barbarus ex. Süd-Afrika

(Süd-Afrika).

Falco barbarus fasciinucha (Rchw. Neum.)

Ost- und Süd-Afrika.

Falco barbarus arabicus Erl. Arabien

(? Nord-Ost-Afrika).

Falco barbarus punicus Lev. Nord-Afrika (Atlasländer).

Falco barbarus babylonicus (L. H. Irby)

Kaukasusgebiet, Babylonien, Euphrat.

Falco barbarus aus Deutschland (entweder eine Brehm'sche

Form oder nov. subspecies germanicus Erl.).

Deutschland, Mitteleuropa.

\section{Falco barbarus barbarus L.}

Abbild.: Schlegel \& Susem. Vögel-Europas Tafel 9 Fig. I. (1839). - Linné S. N. I. pag. 125 (1766). - Falco barbarus. Schlegel \& Susemihl, Vög. Eur. cum Tab. 9. 1839. - Falco peregrinoides Schlegel nec. Temm.

Von diesem Edelfalken, dem echten rotnackigen Berberfalken, liegen mir fünf Exemplare vor und zwar:

đo ad. Nubien, Berl. Mus. No. 1005, leg. Hemprich \& Ehrenberg. Flgl. 28,5, Schwanzl. 15,5, Schnabel 1,9 cm.

\& ad. Nubien, Berl. Mus. No. 1008, leg. Bauerhorst. Flgl. 31,6 , Schwanzl. 17,5 , Schnabel $2,2 \mathrm{~cm}$.

\& ad. (Tring.-Museum) ex Brehm Collection. Januar 1851 Blauer Fluss, Sennar leg. Brehm. Flgl. 32,7, Schwanzl. 17,5, Schnabel $2,2 \mathrm{~cm}$.

đิ ad. (Tring-Museum) ex Brehm Collection. 9. März 1852 Luxor. leg. Alfr. Brehm. Flgl. 27, Schwzl. 15,5, Schnabel 1,9 cm.

Expl. (augenscheinlich ô iuv.) Nubien, Berl. Mus. No. 17608 leg. Wilke. Flgl. 27,2, Schwzl. 15, Schnabel 1,8 cm.

Falco barbarus barbarus hat bei weitem von allen seinen Verwandten das meiste Rot im Nacken, auch die Unterseite ist sehr hell und mit spärlicher Fleckenzeichnung markiert, welche sich an den Hosen und Flanken in eine Querbänderung verwandelt. Zumal die adulten ô $\delta$ haben eine fast einfarbige gelbbraune Unterseite, fast ohne dunkle Zeichnung. Auf der Oberseite, den Flügeln, Schwanz und zumal dem Bürzel ist das Blau sehr hell mit starkem grauem Anflug.

Brutgebiet: Nilländer, Aegypten, Nubien, Sennar.

II. Falco barbarus aus Süd-Afrika event. identisch mit ,fasciinucha."

Temminck Pl. Col. Tab. 479 - Faucon pérégrenoide (adulte mâle) Text. Bd. IV. Falço peregrinoides Temm.

Leider liegt mir kein Exemplar von Falco barbarus ex. Süd-Afrika vor, dagegen ein Exemplar von Falco fasciinucha 
Rchw. Neum. Diagnose Orn. Monatsbr. 1895. pag. 114, ferner noch zwei weitere Exemplare des rotnackigen Wanderfalken aus Arabien, nov. spec., welche ebenfalls dem Falco barbarus ex SüdAfrika sehr nahe stehen.

Reich e now stellt Falco fasciinucha unter die Taubenfalken, eine Ansicht, der ich mich nicht anschliessen kann, der viel stärkere Schnabel, die corpulenteren Läufe und Zehen, die gedrungenern Flügel stempeln ihn sofort als echten Edelfalken.

Temminck gibt in seinem Text, gehörig zur Tafel 479, als Heimat sowohl Nord-Ost-Afrika (Nubien) als auch Süd-Afrika (Meerbusen von Algoa) an. Zu seiner Zeit achtete man nicht auf zoogeographische Formen, und so warf er den rotnackigen Falco barbarus mit dem rotnackigen Berberfalken Süd-Afrikas zusammen. Auf alle Fälle geht daraus hervor, dass auch in Süd-Afrika ein rotnackiger Falke existiert. Aus der Abbildung in Temminck Taf. 479 geht ferner hervor, dass in die Abbildung Characteristica beider zoogeographischen Formen gelegt wurden. Der zu ausgebildete rötliche Nacken, die hellere Unterseite bezieht sich auf nordost-afrikanische Vögel, die scharf ausgeprägte schmale schwarzweisse Bänderung der Unterseite des Schwanzes sind Kennzeichen vom Berberfalken aus Südafrika und zeigen deutlich die nahe Verwandtschaft mit dem ost-afrikanischen Falco fasciinucha Rchw. Neum., der ebenfalls diese praegnante Bänderung auf der Unterseite der Schwanzfedern hat; nur auf der Oberseite, dem Rücken und den Flügeln sehr dunkel ist. Vielleicht fallen auch beide Arten, Falco barbarus fasciinucha Rchw. Neum. und Falco barbarus ex. Süd-Afrika, zusammen.

Brutgebiet: Süd-Afrika.

\section{Falco barbarus fasciinucha Rchw. Neum.}

Orn. Monatsberichte 1895 pag. 114. Falco fasciinucha Rchw. Neum. - Neumann, Journ. f. Orn. 1899. pag. 51. Falco fasciinucha Rchw. Neum. hierzu Taf. 1.

Typus Exempl. (den Massen nach zu urteilen ô ad.) Febr. 95. leg. Neumann, Ndara ('Teita) Berl. Mus. No. 31760. Flgl. $22 \mathrm{~cm}$, Schwzl. 14 ? $\mathrm{cm}$ (an dem ausgestopften Exempl. fraglich), Schnabell. v. d. Wachsh. gem. 1,6 cm.

Schon Neumann spricht im Journ. f. Orn. 1899 pag. 52 von einem Edelfalken, sucht aber dann eine nahe Verwandtschaft bei dem ihm vorliegenden Exemplare mit Falco cuvieri, der doch gar kein Edelfalke ist. Die Folge hiervon ist, dass er auch bei der grossen Suite von Falco cuvieri des brit. Mus. kein Exemplar gefunden hat, welches seinem fasciinucha ähnlich sieht; ein Durchsuchen der Edelfalken dagegen mit rotem Nacken des dortigen Museums, also mit Formen des Formenkreises „barbarus" hätte sicher zu einem andern Resultat geführt.

Dass wir es mit Falco fasciinucha mit einem echten Edelfalken zu tun haben, hat übrigens schon Kleinschmidt richtig erkannt. 
Kurze Betrachtungen über die Gruppe der Edelfalken.

Aquila VIII. 1901 pag. 42 (Anmerkung).

Brutgebiet: Ost- u. Süd-Ost-Afrika.

IV. Falco barbarus arabicus Erl. nov. subsp. Falco barbarus fasciinucha Rchw. Neum. am nächsten stehend.

Auf der Oberseite, dem Oberkopf, Rücken und Oberflügeln heller, mehr grau. Die Bänderung auf der Unterseite der Schwanzfedern mehr verwaschen. Die weniger markierte Bänderung bildet einen $\times$ wunderbaren Übergang zu Falco barbarus barbarus L. Auch die Unterseite mehr rötlich gelb und nicht rotbraun wie bei ost- resp. süd-afrikanischen Vögeln. Von dieser Art liegen mir drei Exemplare vor.

Exempl. ad. Aden Arabien leg. Neumann (Berl. Mus.). Flgl. 27,5, Schwzl. 15,4, Schnabell. v. d. Wachsh. gem. $1,8 \mathrm{~cm}$.

¿ ad. 25. Aug. 99. Arabien (Lahadsch.) leg. Dodson. (Tring. Mus.). Flgl. 27,1, Schwzl. 15,4, Schnabell.v. d.Wachsh. gem. 1,8 cm. Exempl. iuv. Lahadsch (Süd-Arabien) 6. Septbr. 1899 leg. Dodson (Tring. Mus.). Flgl. 26,7, Schwzl. 16, Schnabell. v. d. Wachsh. gem. $1,7 \mathrm{~cm}$.

Brutgebiet: Süd-Arabien (? Nord-Ost-Afrika) nähere Angaben vorerst unmöglich.

V. Falco barbarus punicus (Lev. jun.)

Levaillant jun. Expl. Algérie Ois. Pl. 1. (1850) Falco punicus Levaill. Loche. Expl. scient. de l'Algérie pag. 55 (1867). Gennaia barbarus.

Dieser nord-afrikanische Barbarus-Falke bildet einen wunderbaren Übergang zu dem in Europa vorkommenden „Barbarus". Denn aucb unsere deutschen Wälder bergen als Brutvogel einen Barbarusfalken und besitze ich in meiner Sammlung einen dieser deutschen am Horste erlegten Falken, auf den ich nachher zu sprechen komme.

Falco barbarus punicus hat bei Weitem am wenigsten rot im Nacken. Es ist gewissermassen nur ein Anflug, der auch als Schatten, recht wenig zweckentsprechend und fehlerhaft, in der Abbildung Lev. jun. Pl. 1 angedeutet ist. Dieser rötliche Nackenanflug setzt sich von hier fort, umrahmt die schwarzblauen Backenflecken und gibt den hellen Wangenseiten rötlichen Anflug. Die Unterseite variiert sehr in ihrer rötlichen Färbung, teils auf Alter zurückzuführen, teils auch Übergänge bildend zu Falco barbarus barbarus L., Falco barbarus arabicus Erl, Falco barbarus babylonicus (L. H. Irby) einerseits und dem in Deutschland als Brutvogel vorkommenden, in den Formenkreis „Barbarus“ gehörenden Edelfalken, andererseits. Falco barbarus punicus (Lev. jun.) liegt mir in drei Exemplaren vor.

o ad. Marocco (Rabat) Mai 1898 gek. von Schlüter. Coll. Kleinschmidt. Flgl. 29, Schwanzl. 15, Schnabell. v. d. Wachsh, gem. $1,9 \mathrm{~cm}$. 
Exempl. ad. (anscheinend đే) Käfigvogel (Tring-Mus.). Flgl. 27, Schwanzl. 15,7, Schnabell v. d. Wachsh. gem. $2 \mathrm{~cm}$.

o med. Tanger Marocco (Tring. Mus.). Sehr abgeblasster, alter Balg, Vogel mit sehr zerschlissenem Gefieder. Flgl. 28,2, Schwanzl. 16, Schnabell. v. d. Wachsh. gem. $1,9 \mathrm{~cm}$.

\% iuv. 10. Mai 1898 Aguilas Spanien, (Tring-Mus.) Flgl. 26,5, Schwanzl. 18, Schnabell. v. d. Wachsh. gem. $2 \mathrm{~cm}$.

Brutgebiet: Nord·Afrika, Atlasländer (Tunis, Algier, Marocco). (? Süd-Spanien.)

\section{Falco barbarus babylonicus (L. H. Irby).}

Capt. L. H. Irby On Birds observed in Oudh and Kumaon. Ibis 1861 pag. 218. - Falco babylonicus Irby, hierzu Tafel VII. Von diesem, dem Kaukasus,Terek-Gebiet,Babylonien, EuphratGebiet als Brutvogel angehörenden Barbarusfalken liegt mir ein Exemplar aus der Collection Kleinschmidt vor.

Der Vogel, der an Grösse dem Falco barbarus punicus aus Nord-Afrika gleich kommt, hat noch ausgeprägtere rötliche Nackenfärbung als dieser und bildet einen Übergang in dieser Beziehung zum nubischen Falco barbarus barbarus L., dagegen nähert ihn die mehr gefleckte und mit Wellenlinien versehene Unterseite mehr dem nord-afrikanischen Vogel. Der rötliche Schimmer auf den hellen Wangen, der sich vom rötlichen Nackenbande fortsetzt und die schwarzbraunen Backenstreifen umsäumt, erinnert ebenfalls an F'alco barbarus punicus, jedoch ist letzteres Merkmal zumal bei dem $\$$ \% auch bei Falco barbarus barbarus L. der Fall.

$\$$ ad. 17. Jan. 1900. Kaukasus (Terekgebiet) Prochladnaja. Coll. Kleinschmidt. Flgl. 32,5, Schwanzl. 19, Schnabell. von der Wachsh. gem. $2,8 \mathrm{~cm}$.

VII. Falco barbarus aus Deutschland, subsp. germanicus Erl. oder auf eine Brehm'sche Art zurückzuführen. ? (Brehm Naturg. der Vögel Deutschlands 1831. pag. 62. Falco cornicum, Krähenfalke).

Von dieser Art liegt mir nur ein Exemplar aus meiner Sammlung vor. Der rötliche Anflug im Nacken ist nur angedeutet. Das mir vorliegende 3 unterscheidet sich von den übrigen durch die scharfe dunkel schiefergraue Bänderung auf der Oberseite, zumal praegnant auf dem Bürzel, welcher sich deutlich von dem übrigen blaugrauen, helleren Gefieder abhebt. Auch die dunkle Bänderung am Schwanze ist schmal und ausgeprägt. Die unterste dunkle Binde ebenso schmal wie die übrigen und nicht so breit wie bei den anderen mir aus Deutschland vorliegenden Wanderfalken meiner Sammlung, welche ich zum Formenkreis "peregrinus" gehörig erachte. Die Unterseite ist nicht völlig mit schwarzen Längsstrichen geziert, welche sich bindenartig fortsetzen, sondern ist nur an den Seiten gebändert, während in der Mitte der Unterseite eine deutliche dunkle Fleckung vorhañden ist. 
Der Schnabel ist auf der First breiter, überhaupt gedrungener und dicker. Die Flügel kürzer.

Brehm spricht in seinen Werken immer von zwei Arten, dem Falco cornicum und Falco peregrinus. In seiner Naturgeschichte der deutschen Vögel, pag. 62, 63, verweist er auf Naumann's Naturgeschichte der Vögel Deutschlands. Tafel 24, 1; 25,$2 ; 24,2 ; 25,1$. Naumann citiert die Bilder als GeschlechtsUnterschiede. Die beiden alten Vögel zeigen deutlich den Unterschied der Befiederung auf der Unterseite.

Was die Grössenunterschiede anbelangt, von welchen Brehm in seiner Naturgeschichte der deutschen Vögel spricht, pag. 63, welche zwischen beiden Arten bestehen, ferner die osteologischen Unterschiede sind entschieden beachtenswert. Mir fehlt es an Material, um auf diese Fragen näher einzugehen.

Auf alle Fälle hat mein Exemplar, welches ich zum Formenkreis „Barbaru s“ gehörig erachte, kürzere Flügel und dickeren Schnabel als die übrigen $\mathrm{mir}$ vorliegenden Wanderfalken aus Deutschland. Auch die Stirn ist bei diesem deutschen Barbarusfalken mehr graublau, die Federn heller umsäumt und nicht wie bei den Peregrinus-Falken tief dunkel schieferblau, welches sich im Nacken fortsetzt.

đ ad. 15. April 1899. Deutschland, Heldra bei Treffurt, leg. Förster Menzel Coll. v. Erlanger. Flügel 28.9, Schwanzl. 14,5, Schnabell. v. d. Wachsh. gem. 1,8, Oberschnabeldicke gem. v. Dornfortsatz zu Dornfortsatz $0,7 \mathrm{~cm}$.

Aus dem Formenkreis "Peregrinus" Tunst., communis Gm., liegen mir auch mehrere zoogeographischen Formen vor; jedoch genügt mir mein Material nicht, ebenso wie es noch viele andere Formen der beiden anderen Formenkreise gibt, so auch von diesem. Ich will mich daher darauf beschränken, nur zoogeographische Formen zu erwähnen, bespreche die Masse des mir vorliegenden Materials und überlasse andern Ornithologen an der Hand noch grösseren Materials darauf zurückzukommen. Wie überhaupt meine Arbeit nur eine Anregung bilden soll, ein Stückwerk ist, vielleicht eine falsche Auffassung!

Folgende zoogeographische Formen des Formenkreises „,Peregrinus" sind mir bekannt und liegen mir vor.

I. Falco peregrinus peregrinus $\mathrm{Gm}$. aus Deutschland, Mittel-Europa.

II. Falco peregrinus brittanicus Erl. England, Schottland.

III. Falco peregrinus griseiventris (Brehm) aus Schweden, Nordrussland.

IV. Falco peregrinus anatum (Bp.) aus Grönland.

V. Falco peregrinus atriceps aus Süd-Russland, Persien, NordIndien.

VI. Falco peregrinus leucogenys (Brehm) aus Süd-Ost-Europa, Süd-West-Asien.

VII. Falco peregrinus brookii Sharpe aus der Mittelmeer-Subregion, 
VIII. Falco peregrinus peregrinoides Smith, Süd-Afrika nördlich bis zum Congo und Nyassa-See.

IX. Falco peregrinus radama (Hartl.) aus Madagascar.

I. Falco peregrinus peregrinus (Gm.). Gmelin. S. N. I. pag. 272, (1788 Briss.) - Falco peregrinus Gm. 1788 ex Buff. Falco communis Gm. - Brehm. Naturg. der deutschen Vögel 1831 pag. 62 Falco peregrinus L. (Falco abietinus Bechst.). Brutgebiet: Mittel-Europa, (Deutschland).

Characteristica für diesen Wanderfalken sind die tief schwarze Stirn, Kopfplatte und Nacken, die gelbbraune Unterseite, die von der Brust abwärts mit schwarzen Längsflecken, Wellenlinien bildend geziert ist, verändert sich auf den Flanken und Hosen in schmutzig Grauweiss. Oberseite, Rücken, Flügel, vom Nacken anfangend sich verhellend bis blaugrau mit undeutlich dunkler Wellenzeichnung, welche nur auf den oberen Schwanzdeckeln praegnant zum Vorschein tritt.

Aus meiner Collection liegen mir von dieser zoogeographischen Form 6 Exemplare vor, deren Masse folgende sind.

\$ ad. Hannover 10. Okt. 98 erlegt von Förster Frühauf. Flg. 29,7, Schwzl. 16,2, Schnabel v. d. Wachsh. gem. 1,9, Oberschnabel v. Dornfortsatz zu Dornfortsatz $0,6 \mathrm{~cm}$.

ठ́ ad. Hannover 7. April 99 erlegt von Förster Frühauf. Flgl. 29,9, Schwzl. 16,2, Schnab. v. d. Wachsh. gem. 1,8 cm.

o Westpreussen Heldra bei Treffurt erlegt von Förster Menzel 12. April 99. Flgl. 29,8, Schwzl. 16, Schnabel v. d. Wachsh. gem. 1,8, Oberschnabel von Dornfortsatz zu Dornfortsatz $0,6 \mathrm{~cm}$.

$\$$ ad. Hessen (Nieder-Ingelheim) 12. Februar 99 leg. Hilgert. Flgl. 35,3, Schwanzl. 20, Schnabel v. d. Wachsh. gem. $2,3 \mathrm{~cm}$.

$\%$ ad. Ostpreussen, (Oszywilken bei Arys) erl. von Förster Vaternam) 1. Juli 99. Flgl. 34,7, Schwanzl. 19, Schnabel v. d. Wachsh. gem. $23 \mathrm{~cm}$.

\% ad. Ostpreussen, (Oszywilken) 3 Jahre in der Gefangenschaft. 1. Februar 1902 verendet. Flgl. 35,9, Schwanzl. 20, Schnabel v. d. Wachsh. gem. $2,5 \mathrm{~cm}$.

II. Falco peregrinus brittanicus Erl. nov. subspecies.

(fragliche Form).

Dieser englische Wanderfalke unterscheidet sich von mitteleuropäischen (Form I) und nordischen (Form III), indem er in der Mitte zwischen beiden zoogeographischen Formen steht. Falco peregrinus brittanicus hat die Unterseite wie der deutsche Wanderfalke, vielleicht um ein Atom heller, zumal scheint die graue Grundfarbe auf Flanken und Hosen im allgemeinen reiner weiss $\mathrm{zu}$ sein.

Die Oberseite dagegen ist heller als bei Falco peregrinus peregrinus und entspricht an Helle der von Falco peregrinus 
griseiventris. Zumal tritt dieser Unterschied zwischen Form I und II deutlich auf Stirn, Kopfplatte und Backenstreifen zu Tage.

Der brittanische Wanderfalke liegt mir in 7 Exemplaren vor.

o Jolly Farm near Tring, England, Herts. (Tring Mus.). 4. Oktbr. 97. Flgl. 30,2, Schwzl. 17, Schnabel v. d. Wachsh. gem. $2,1 \mathrm{~cm}$.

t ad. Isle of Man (Tring Mus.). Flgl. 30, Schwzl. 16,1, Schnabel v. d. Wachsh. gem. $1,9 \mathrm{~cm}$.

$\%$ ad. England Isle of Man (Tring. Mus.). Flgl. 34,5, Schwzl. 20,3, Schnabel v. d. Wachsh. gem. $2,5 \mathrm{~cm}$.

$\%$ ad. Lungwell, Gutherlund, England 6. VIII. 73 (Tring. Mus.). Flgl. 33,3, Schwzl. 19,5, Schnabel v. d. Wachsh. gem. 2,5 cm.

$\delta$ ad. N. W. Schottland 25. Mai, Tring. Mus. Flgl. 30,6, Schwzl. 16,5, Schnabel v. d. Wachsh. gem. $2 \mathrm{~cm}$.

\% ad. North Berwick, Schottland 18. Dezbr. 96 (Tring. Mus.). Flgl. 34,5, Schwzl. 21, Schnabel v. d. Wachsh. gem $2,5 \mathrm{~cm}$.

Letztere beiden schottischen Exemplare nähern sich mehr schwedischen Vögeln, während die englischen Falken sich mehr mitteleuropäischen Vögeln nähern. Entschieden bilden aber die Wanderfalken Brittaniens eine Zwischenform zwischen I und III. Dieselben kommen in ihren Massen schwedischen Vögeln gleich, zumal bei den $\hat{\sigma}^{*} \hat{\sigma}^{*}$ ist der Schnabel im allgemeinen stärker als bei mitteleuropäischen Vögeln, was aus obigen Massen hervorgeht.

\section{Falco peregrinus griseiventris (Brehm).}

Brehm Isis 1833 pag. 778 Falco griseiventris Brehm. Typus (Tring. Mus.) ex Brehm Collection.

o erl. v. Baron v. Beust 24. Okt. 1826. Nimritz (Neustadt Orla).

(Das Typus-Exemplar trägt ausser der Museumsetikette die Originaletikette von Brehm). Characteristica dieser zoogeographischen Form sind:

Die hellgraue Unterseite, welche nach den Flanken, Bauche und Hosen zu schmutzig grauweiss ist. Die ganze Unterseite ist mit schmalen dunklen Längsflecken geziert, welche sich in Wellenlinien an einander reihen. Oberseite, Rücken, Bürzel, Oberschwanzdecken, Oberseite der Flügel sehr lichtgrau. Auch Stirn, Oberkopf, Nacken und Backenflecken haben auf dem Schwarz grauen Anflug, der zumal bei den đే ô stark hervortritt. $\$$ \% haben auf der hellen Unterseite gelblichen Anflug. Schnabel sehr stark, zumal bei den $\delta$ o.

Brutgebiet: Schweden, (? Norwegen) Nordrussland.

Es liegen mir ausser dem Typus 6 Exemplare des Tring. Mus. vor und 1 Exemplar aus meiner Collection.

đ ad. Göteborg, Schweden 25. IV. 1901, Tring. Mus. Flgl. 31,4, Schwzl. 16,1, Schnabel v. d. Wachsh. gem. $2 \mathrm{~cm}$.

to ad. Haburgen, Schweden 18. V. 1834 ex Brehm Collection (Tring. Mus.). Flgl. 30,2, Schwzl. 17, Schnabel v. d. Wachsh. gem. $2 \mathrm{~cm}$. 
đ ad. Göteborg, Schweden 10. VII. 1900, Tring. Mus. Flgl. 31,7 , Schwzl. 17, Schnabel v. d. Wachsh. gem. $2 \mathrm{~cm}$.

o ad. Gouvernement St. Petersburg, Nordrussland 23. VI. 1900. Coll. v. Erlanger. Dieses Exemplar wurde auf einem freien Hochmoor am Horst, der zwei Junge enthielt, von Freiherrn von Osten-Sacken im Eisen gefangen. Flgl. 30,8, Schwzl. 16,3 , Schnabel v. d. Wachsh. gem. $1,8 \mathrm{~cm}$.

$\$$ ad. Göteborg, Schweden 16. I. 01. (Tring. Mus.). Flgl. 34,8 , Schwzl. 19,1, Schnabell. v. d. Wachsh. gem. $2,5 \mathrm{~cm}$.

\% Göteborg, Schweden 28. IX. 1901 (Tring. Mus.). Flgl. 34,6, Schwzl. 20, Schnabell. v. d. Wachsh. gem. 2,4 cm.

\% ad. Göteborg, Schweden 3. V. 1901 (Tring. Mus.). Flgl. 34,6 , Schwzl. 20, Schnabl. v. d. Wachsh. gem. $2,2 \mathrm{~cm}$.

Das $\delta$ aus Nordrussland, Gouv. st. Petersburg, aus meiner Sammlung hat auffallend kleinen Schnabel, 1,8 cm; während die übrigen $\delta$ o dieser Art aus Schweden alle $2 \mathrm{~cm}$ haben. Es nähert sich daher seinem südrussischen Verwandten.

\section{Falco peregrinus anatum (Bp.).}

Bonaparte Comp. List. B. Eur. N. Am. pag. 4. (1838 ex Adubon).

Das Brutgebiet dieses schönen Wanderfalken ist Grönland. Oberseite sehr dunkel schwarzblau, auf der Unterseite isabellfarben mit schwarzer Wellenzeichnung.

Falco peregrinus anatum liegt mir nur in einem Exemplar vor.

đ II. Herbst (einmal vermausert) Holstenborg, Grönland. 5. Okt. 1898 Coll. Kleinschmidt. Flgl. 31,2, Schwzl. 18, Schnabl. v. d. Wachsh. gem. $1,9 \mathrm{~cm}$.

\section{Falco peregrinus atriceps (Hume).}

Hume Ibis 1869 pag. 356 Falco atriceps Hume.

Dem Falco peregrinus griseiventris am nächsten, unterscheidet sich von diesem durch die dunklere Oberseite, welche zumal auf Oberkopf, Nacken und Backen stark hervortritt und den grauen Schimmer des schwedischen resp. nordrussischen Wanderfalken entbehrt. Ferner unterscheidet sich diese Art von Form III durch den meist kleinen Schnabel. (Zwar nicht constant).

Die Unterseite ist reiner weiss wie bei den ô ôे von Form III, die gelbliche Unterseite der $\$$ \% ebenfalls heller als bei Form III, zuweilen auch reinweiss. Auch ist die dunkle Wellenzeichnung viel spärlicher und öfter durch vereinzelte Flecken zumal nach der Mitte der Unterseite $\mathrm{zu}$ ersetzt. Diese Art liegt $\mathrm{mir}$ in 5 Exemplaren vor.

Genau das Brutgebiet anzugeben ist mir vorerst unmöglich. Südrussland? Persien, Nordindien.

Zugvogel 9. Januar 1900. Flgl. 36, Schwzl. 21, Schnabel v. d. Wachsh. gem. $2,2 \mathrm{~cm}$. 


\section{Falco peregrinus leucogenys (Brehm).}

Brehm Naumannia 1855 pag. 268 Falco leucogenys.

Brutgebiet: Süd-Ost-Europa, Süd-West-Asien.

Unterscheidet sich von seinen Verwandten durch die bedeutende Grösse und blassere Färbung auf der Oberseite.

\& ad. Montenegro, albanisches Territorium 10. II. 1900 leg. v. Führer (Tring. Mus.). Flgl. 36,5, Schwzl. 20,5, Schnabl. v. d. Wachsh. gem. $2,4 \mathrm{~cm}$.

O iuv. Montenegro 16. II. 1900 leg. v. Führer (Tring. Mus.). Flgl. 36, Schwzl. 20,5, Schnabel v. d. Wachsh. gem. $2,3 \mathrm{~cm}$. Erlanger.

\& ad. Zeyla, Nordküste des Somalilandes. Collection von

đิ ad. Petrowsk Süd-Russland 17. V. 95. Coll. v. Erlanger. Flgl. 30, Schwzl. 17, Schnabel v. d. Wachsh. gem. 1,9 cm.

o ad. Madras, Indien, Zugvogel, Tring. Mus. Flgl. 30,7 Schwzl. 18, Schnabel v. d. Wachsh. gem. $2 \mathrm{~cm}$.

đad. Madras, Indien, Zugvogel, Tring. Mus. Flgl. 29, Schwzl. 18, Schnabel v. d. Wachsh. gem. $1,9 \mathrm{~cm}$.

$\$$ ad. Behemba, Nord-Persien Februar 95 (Tring. Mus.). Flgl. 34, Schwzl. 20, Schnabel v. d. Wachsh. gem. 2,3 cm.

Nach Kleinschmidt sind die Brutvögel des Himalaya und Süd-Indiens blutrot auf der Unterseite, was er an Brutexemplaren des britischen Museums zu constatieren Gelegenheit hatte; bilden also eine andere zoogeographische Form.

\section{Falco peregrinus brookii (Sharpe).}

Sharpe Ann. N. H. (4) XI. pag. 20, 222. (1873). Falco brookii.

Von dieser Wanderfalkenart, dessen Brutgebiet die Mittelmeer-Subregion ist, liegen mir 5 Exemplare vor. Es ist der kleinste palaearctische Wanderfalke mit dunkler Oberseite, zumal auf Kopf und Nacken. Die Unterseite scheint auch einer gewissen individuellen Variation zu unterliegen, wenigstens ist dies meine vorläufige Ansicht, da ich vorerst nicht annehme, dass der Wanderfalke Sardiniens und Corsikas als insuläre Form betrachtet werden muss. Bei 2 Exemplaren aus Tanger und einem aus Süd-Spanien hat nämlich die Unterseite unter dunkler, häufig gewellter Zeichnung gelblichen Anflug, während die bei den $\mathbf{9} \mathbf{9}$ sehr dunkle und wellenreiche Zeichnung der Unterseite, sowie die des dazu gehörigen ơ aus Sardinien auf grauweissem Grundton liegt.

$\delta$ ad. Aguilas, Süd-Spanien. 12. IV. 99 (Tring. Mus.). Flgl. 28, Schwzl. 14,7, Schnabel v. d. Wachsh. gem. $1,9 \mathrm{~cm}$.

$\$$ ad. Tanger, Marocco 7. V. 94 (Tring. Mus.). Flgl. 32,2, Schwzl. 18, Schnabel v. d. Wachsh. gem. $2,2 \mathrm{~cm}$.

\% ad. Tanger Marocco 14. V. 85 (Tring. Mus.). Flgl. 32, Schwzl. 17,6, Schnabel v. d. Wachsh. gem. $2,2 \mathrm{~cm}$.

o ad. (Sassari, Nurra die Torres), Sardinien 7. VI. 1902. Coll. v. Erlanger. Flgl. 28,5, Schwzl. 15, Schnabel v. d. Wachsh. gem. $1,9 \mathrm{~cm}$. 
\% ad. Eliastra Bellavista, Sardinien 17. X. 1902. Coll. v. Erlanger. Flgl. 32,3, Schwzl. 18, Schnabel v. d. Wachsh. gem. $2,3 \mathrm{~cm}$.

\section{Falco peregrinus peregrinoides (Smith).}

Smith S. Afr. Q. Journ. 1. pag. 235, (1830) Falco peregrinoides Smith).

Sharpe Cat. of Birds. Britt. Museum I pag. 383 cum. Tab. 12 Falco minor Bp. Diese Art liegt mir in drei Exemplaren vor.

đ ad. Songea, Tanganyikagebiet leg. Stierling 10. IV. 1900. (Kgl. Mus. Berlin). Flgl. 18,7, Schwzl. 15, Schnabel. v. d. Wachsh. gem. 1,9 cm.

Expl. ad. leg. Stanley-Pool, Congo. (Tring. Mus.). Flgl. 18, Schwzl. 15, Schnabel v. d. Wachsh. gem. 1,8 cm.

o ad. Sansibar (Tring. Mus.). Flgl. 17,3, Schwanzl. 14,3 Schnabel v. d. d. Wachsh. gem. $1,9 \mathrm{~cm}$.

\section{Falco peregrinus radama (Hartl.).}

Hartlaub Faun. Madag. 1861 pag. 17 Falco radama Hartlaub.

François P. L. Pollen und D. G. van Dam Fauna Madag. Ois. 1868 pag. 31 Falco communis.

Hartlaub. Die Vögel Madagaskars 1877 pag. 10 Falco minor Bp.

Falco radama Hartlaub, der Falco peregrinoides Smith sehr ähnlich sieht und ihm am nächsten von seinen übrigen Verwandten steht, unterscheidet sich von letzterem erstens durch die viel dunklere Oberseite, nämlich Oberkopf, Nacken und Rücken. Während sich bei Falco peregrinoides die hellgraue Färbung der unteren Hälfte des Rückens, Bürzels und der Oberschwanzdecken deutlich von der dunkleren, sammtschwarzen oberen Hälfte des Rückens, des Nackens und Kopfes abhebt, so bedeckt diese dunkle Färbung die ganze Oberseite der Madagaskar-Wanderfalken. Dieses dunkle Aussehen wird noch dadurch verstärkt, dass die gebänderte dunkle Wellenzeichnung der Oberseite zumal auf dem Bürzel und den Oberschwanzdecken deutlich hervortritt. Auch die Flügeldeckfedern sind sehr dunkelschwarz. Die schwarze Bänderung der Unterseite ist auf gelblichem Grundton viel breiter und intensiver, während sie bei den Wanderfalken des tropisch-afrikanischen Continents schmaler und zumal auf den Flanken und Hosen auf grauem Grundton verwaschener ist.

Sowohl aus dem Formenkreis „Barbarus" wie „Peregrinus" gibt es noch eine ganze Beihe anderer Formen, jedoch sind diese teils noch völlig unbekannt, teils genügt das mir vorliegende Material nicht, um richtig erkannt zu werden. Falco ernesti Sharpe aus Brit. New. Guinea, von dem mir ein Exemplar des 
Tring Mus. vorliegt. Ferner Falco peregrinus aus Labrador und Mexiko, event. identisch mit Falco peregrinus anatum, aus Grönland u. s. w., gehören ebenfalls zu diesem Formenkreis, doch würde es mich zu weit führen, meine Arbeit über Europa, Asien und Afrika hinaus auszudehnen.

Ein dem Tring. Mus. gehöriger Edelfalke aus Fuerte-Ventura bildet event. auch eine zoogeographische Form des Formenkreises „Barbarus,“ jedoch liegt mir von dort nur dieser eine Falke vor. Eine offene Frage! Zu welchem Formenkreis gehört der australische Falco melanogenys Gould und der Edelfalke aus Japan.

Vor allem mein aufrichtigsten und ergebensten Dank Herrn Baron von Rothschild, der mir in liebenswürdigster und zuvorkommendster Weise das bedeutende Material von Edelfalken aus dem Tring Museum zur Verfügung gestellt und zum Vergleich mit dem Material des Kgl. Mus. in Berlin hierher gesandt hat.

Herrn Kleinschmidt verdanke ich einige prachtvolle Bälge seiner Collection, die mir ebenfalls zum Vergleich vorlagen.

\section{Dentsche Ornithologische Gesellschaft. Bericht über die Novembersitzung.}

Verhandelt Berlin, Montag, den 3. November 1902, Abends $8 \mathrm{Uhr}$ im Architekten-Vereinshause, Wilhelmstrasse 92. II.

Anwesend die Herren: Schalow, Reichenow, Deditius, Thiele, von Treskow, Paeske, O. Neumann, Jacobi, Heinroth, Freiherr von Erlanger, Schillings, Matschie, Haase.

Als Gast: Herr G. Haase.

Vorsitzender: Herr Schalow. Schriftf.: Herr Matschie.

Im Anschluss an die Vorlegung neuer ornithologischer Schriften durch die Herren Reichenow und Matschie erhebt sich über einige zur Erörterung gelangte Fragen eine lebhafte Besprechung.

Über das Brutgeschäft von Struthio massaicus äussern sich die Herren Schillings, Neumann und Freiherr von Erlanger.

Herr Reichenow empfiehlt, dass durch unsere Vogelwarte in Rossitten eine ähnliche Zusammenstellung der Brutplätze von Ciconia alba für Ostpreussen gemacht werde, wie sie für Mecklenburg jetzt durch Wüstnei vorliegt.

Herr Schalow weist auf die zahlreichen, nicht zur Brut gelangenden Störche hin und vermutet, dass diese abgeschlagene alte Männchen sind. 


\section{$2 \mathrm{BHL}$ Biodiversity Heritage Library}

1903. "Kurze Betrachtungen über die Gruppe der Edelfalken." Journal fu

r Ornithologie 51, 289-301. https://doi.org/10.1007/bf02207107.

View This Item Online: https://www.biodiversitylibrary.org/item/104726

DOI: https://doi.org/10.1007/bf02207107

Permalink: https://www.biodiversitylibrary.org/partpdf/141621

\section{Holding Institution}

Smithsonian Libraries

\section{Sponsored by}

Biodiversity Heritage Library

\section{Copyright \& Reuse}

Copyright Status: Public domain. The BHL considers that this work is no longer under copyright protection.

This document was created from content at the Biodiversity Heritage Library, the world's largest open access digital library for biodiversity literature and archives. Visit BHL at https://www.biodiversitylibrary.org. 